\title{
Three-Phase Switch-Mode Rectifier for Y-Connected Sources with an Unaccessible Neutral
}

\author{
Yu-Kang Lo, Wen-Tsair Li \\ Department of Electronic Engineering \\ National Taiwan University of Science and Technology \\ Taipei, Taiwn, ROC \\ yklo@et.ntust.edu.tw
}

\begin{abstract}
A three-phase switch-mode rectifier (SMR) suitable for three-wire Y-connected sources with an unaccessible neutral is proposed in this paper. Each phase current is split into two line currents. By controlling the line currents to be sinusoidal and in-phase with the corresponding line-to-line voltages, unity input power factor for each phase can be obtained. The access of the neutral for command reference is no longer required. Only three active switches are utilized. Also there is no need to insert the dead times for preventing the simultaneous conduction of the switches. Simulations and experiments on a prototype show satisfactory results.
\end{abstract}

\section{Introduction}

Three-phase switch-mode rectifiers (SMR's) are widely used in high power $\mathrm{AC} / \mathrm{DC}$ applications where the harmonic contents of input currents are confined. In the literature [1-3], many circuit topologies are presented to achieve unity power factor. Most of the three-phase sources are Yconnected for the concern of safety. To sense the reference waveform of each phase voltage for the phase current to follow, the access of the neutral is necessary. However, in a three-phase threewire Y-connected system, sometimes it is difficult to get the neutral. In this paper, we adopt a three-phase SMR composed of three modified single-phase boost SMR's[3], as shown in Fig. 1, for the Y-connected sources with an unaccessible neutral. The power circuits in dotted boxes are identical. The outputs of the three single-phase SMR's are parallel connected to the same load. Compared to the standard single-phase one-switch SMR, the modified boost circuit is added with a second input inductor and a second fast-recovery diode. For example, to prevent $i_{\mathrm{ab}}$ from flowing into the negative pole of the load and causing interactions with other two phases when $S_{a b}$ is turned on, $D_{a b}^{\prime}$ is inserted in the path between $S_{a b}$ and the load. To ensure that $i_{b a}$ will always remain continuous, a second input inductor $L^{\prime}$ ab is added as shown in Fig. 1. Thus when $S_{a b}$ is turned off, $D^{\prime}{ }_{a b}$ will be forced to turned on to form a conduction path for $i_{\text {ba }}$.

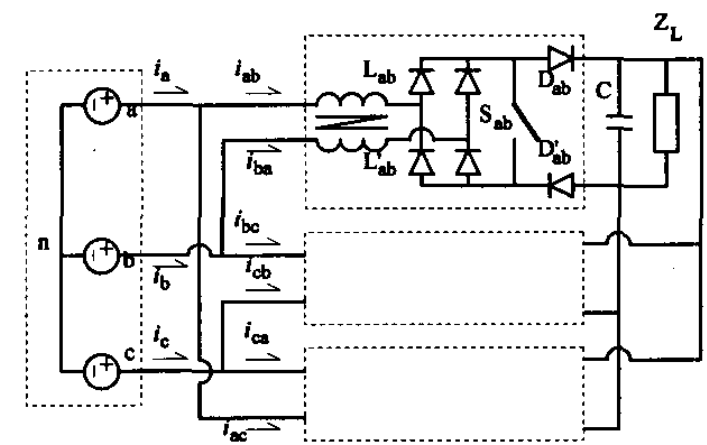

Fig. 1 The proposed three-phase SMR for 3-wire Y-connected sources.

In the proposed three-phase SMR, each phase current is split into two line currents. By controlling the line currents to be sinusoidal and in-phase with the corresponding line-to-line voltages, unity input power factor for each phase can be obtained. The access of the neutral for command reference is no longer required. Only three active switches are utilized. The rectifier circuit for each phase is operated independently. There is no need to insert the dead times for preventing the simultaneous conduction of the switches. Although the decoupling among three phases can be violated when two or more switches are simultaneously turned off, the effects of the interactions are negligible by using coupled inductors instead for each single-phase SMR. Simulations and prototype experiments show satisfactory results. 


\section{Circuit Description}

In a three-phase three-wire Y-connected system, we can only sense the scaled waveforms of lineto-line voltages through the transformers. Of course the referenced waveforms of the phase voltages can be synthesized for the phase currents. For example, assuming that $n$ ' is the common ground of the secondary side of the transformers:

$v_{\mathrm{abn}}+v_{\mathrm{acn}},=v_{\mathrm{ann}}-v_{\mathrm{ban}}+v_{\mathrm{ann}}-v_{\mathrm{can}}=3 v_{\mathrm{ann}} \mathrm{n}^{\prime}$

where $v_{i j p}$, means a waveform bearing the same magnitude and phase with $v_{i j}$ but with the zero potential at n'. The phasor diagram of the referred voltages is shown in Fig. 2.

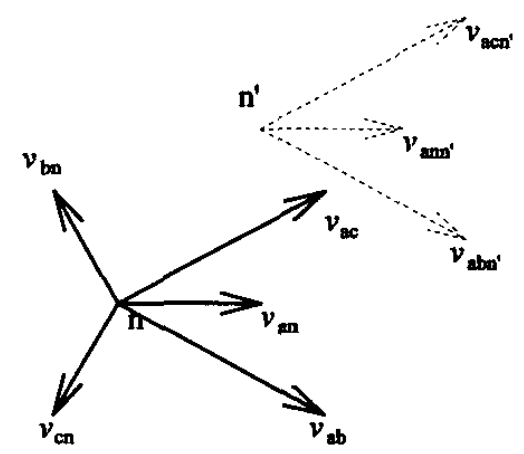

Fig. 2 The phasor diagram of the referred voltages.

However, at least three analog adders are required to obtain the three phase voltages for reference. These sensitive IC's must be tuned with high accuracy to provide an adequate performance. As shown in Fig. 1 , we split the phase current $i_{\mathrm{a}}$ into two components $i_{\mathrm{ab}}$ and $i_{\mathrm{ac}}$, which are respectively the line currents of $v_{\mathrm{ab}}$ and $v_{\mathrm{ca}}$ loops. According to (1), if the split line current can be controlled to follow the corresponding line-to-line voltage, the phase current will also resemble the phase voltage. Thus unity power factor for each phase can be achieved without access of the neutral. To simplify the discussions, here we draw the $v_{\mathrm{ab}}$ loop in Fig. 3(a) and assume that the two input inductances are equal. Considering $v_{\mathrm{ab}}$ is positive, when $S_{a b}$ is turned on, the inductors are charged through $v_{\mathrm{ab}}$ as shown in Fig. 3(b) In this case the $v_{a b}$ loop is completely decoupled from the other two loops. And the changes of $i_{\mathrm{ab}}$ and $i_{\mathrm{ba}}$ depend on the polarity and the magnitude of $v_{\mathrm{ab}}$ alone. Thus:

$i_{\mathrm{ab}}=-i_{\mathrm{ba}}$, and $\frac{\mathrm{d} i_{\mathrm{ab}}}{\mathrm{dt}}=-\frac{\mathrm{d} i_{\mathrm{ba}}}{\mathrm{dt}}=v_{\mathrm{ab}} / \mathrm{L}$

The conclusion made above applies to the other two loops. On the other hand, when $S_{a b}$ is turned off, the conduction path for $v_{\mathrm{ab}}$ loop is as shown in Fig. 3(c). If the switches of the other two loops are both turned on and thus these two loops are decoupled, the changes of $i_{\mathrm{ab}}$ and $i_{\mathrm{ba}}$ depend on the polarity and the magnitude of $\left(v_{a b}-V_{0}\right)$ alone. That is:

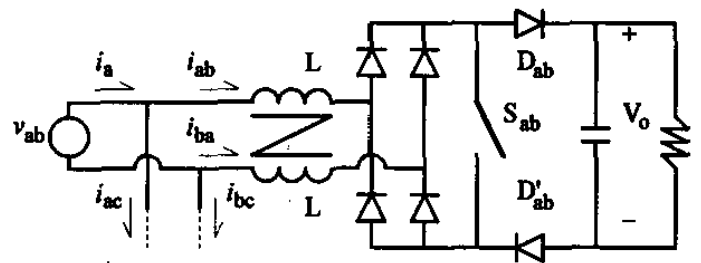

(a)
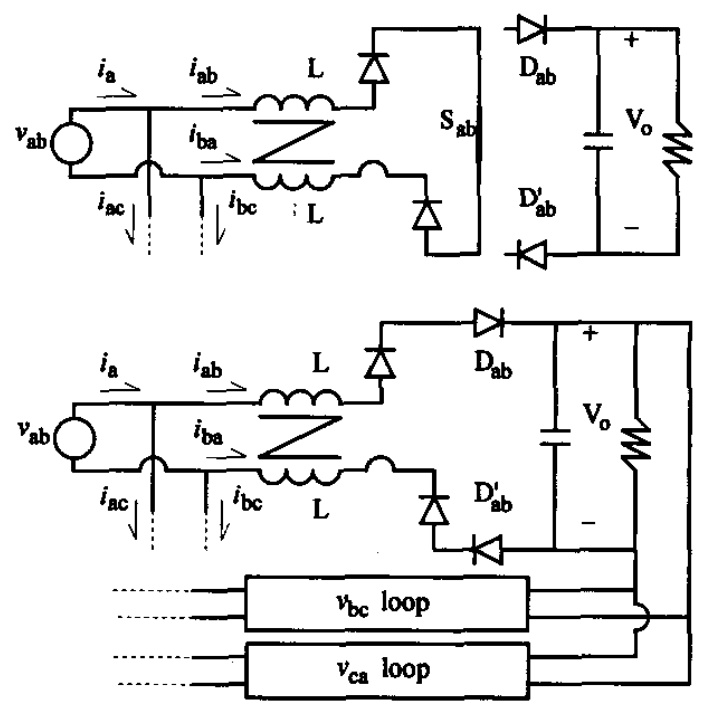

(c)

Fig. 3(a) The $v_{a b}$ loop. (b) The conduction path when $S_{\mathrm{ab}}$ is turned on. (c) The conduction path when $S_{a b}$ is turned off.

$i_{\mathrm{ab}}=-i_{\mathrm{ba}}$, and $\frac{\mathrm{d} i_{\mathrm{ab}}}{\mathrm{dt}}=-\frac{\mathrm{d} i_{\mathrm{ba}}}{\mathrm{dt}}=\left(v_{\mathrm{ab}}-\mathrm{V}_{\mathrm{o}}\right) / \mathrm{L}$

However, if one or both of the other two switches, $S_{b c}$ and $S_{c b}$ is also turned off, then the decoupling among the three loops will no longer sustain. To state the situations more clearly, the 
analysis will be made under the circumstances that $v_{\mathrm{ab}}, v_{\mathrm{bc}}$ and $v_{\mathrm{ca}}$ are all positive as follows.

I. $S_{\mathrm{ab}}$ and $S_{\mathrm{bc}}$ are turned off, and $S_{\mathrm{ca}}$ is turned on:

The conduction paths for the three loops are shown in Fig. 4. It can be observed that $v_{\mathrm{ca}}$ loop is independent from the other two loops. Thus:

$i_{\mathrm{ca}}=-i_{\mathrm{ac}}$, and $\frac{\mathrm{d} i_{\mathrm{ca}}}{\mathrm{dt}}=-\frac{\mathrm{d} i_{\mathrm{ac}}}{\mathrm{dt}}=v_{\mathrm{ca}} / \mathrm{L}$

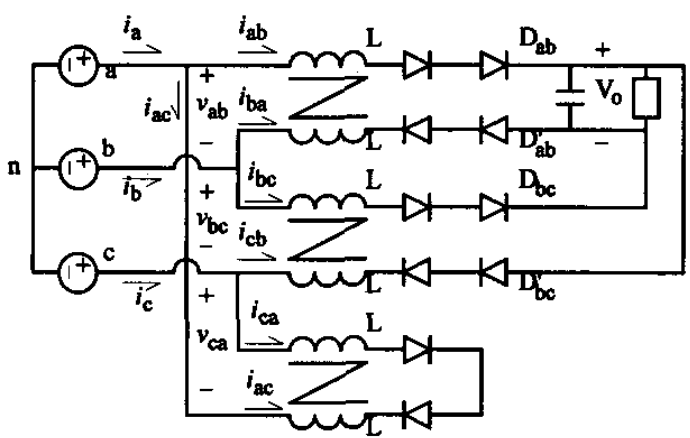

Fig. 4 The conduction paths when $S_{a b}$ and $S_{b c}$ are turned off, and $\mathrm{S}_{\mathrm{ca}}$ is turned on.

As for $v_{\mathrm{ab}}$ and $v_{\mathrm{bc}}$ loops, they are affected by each other because of their outputs are tied together. According to Fig. 4, some equations can be deduced as follows.

$i_{\mathrm{ab}}+i_{\mathrm{ba}}+i_{\mathrm{bc}}+i_{\mathrm{cb}}=0$, and

$\frac{\mathrm{d} i_{\mathrm{ab}}}{\mathrm{dt}}+\frac{\mathrm{d} i_{\mathrm{ba}}}{\mathrm{dt}}+\frac{\mathrm{d} i_{\mathrm{bc}}}{\mathrm{dt}}+\frac{\mathrm{d} i_{\mathrm{cb}}}{\mathrm{dt}}=0$

$v_{\mathrm{ab}}=\mathrm{L} \frac{\mathrm{d} i_{\mathrm{ab}}}{\mathrm{dt}}+\mathrm{V}_{\mathrm{o}}-\mathrm{L} \frac{\mathrm{d} i_{\mathrm{ba}}}{\mathrm{dt}}$

$v_{\mathrm{bc}}=\mathrm{L} \frac{\mathrm{d} i_{\mathrm{bc}}}{\mathrm{dt}}+\mathrm{V}_{\mathrm{o}}-\mathrm{L} \frac{\mathrm{d} i_{\mathrm{cb}}}{\mathrm{dt}}$

From (5), (6) and (7), we can obtain:

$$
\begin{aligned}
& \frac{\mathrm{d} i_{\mathrm{ab}}}{\mathrm{dt}}=\left(3 v_{\mathrm{ab}}+v_{\mathrm{bc}}-2 \mathrm{~V}_{\mathrm{o}}\right) / 4 \mathrm{~L} \\
& -\frac{\mathrm{d} i_{\mathrm{be}}}{\mathrm{dt}}=\left(v_{\mathrm{ab}}-v_{\mathrm{bc}}-2 \mathrm{~V}_{\mathrm{o}}\right) / 4 \mathrm{~L} \\
& \frac{\mathrm{d} i_{\mathrm{bc}}}{\mathrm{dt}}=\left(-v_{\mathrm{ab}}+v_{\mathrm{bc}}-2 \mathrm{~V}_{\mathrm{o}}\right) / 4 \mathrm{~L} \\
& -\frac{\mathrm{d} i_{\mathrm{c}}}{\mathrm{dt}}=\left(v_{\mathrm{ab}}+3 v_{\mathrm{bc}}-2 \mathrm{~V}_{\mathrm{o}}\right) / 4 \mathrm{~L}
\end{aligned}
$$

We can see that the change rates of the two split line currents in $v_{\mathrm{ab}}$ and $v_{\mathrm{bc}}$ loops are different.
Since only one of the two line currents can be sensed in each loop for the feedback signal, it could happen that the other line current runs out of the hysteresis band. Thus distortion may be produced in the phase current, which is the sum of two line currents.

\section{II. $S_{\mathrm{ab}}, \mathrm{S}_{\mathrm{bc}}$ and $\mathrm{S}_{\mathrm{ca}}$ are turned off:}

The conduction paths for the three loops in this case are shown in Fig. 5 . It can be concluded that the change rates of the two split line currents in $v_{\mathrm{ab}}, v_{\mathrm{bc}}$ and $v_{\mathrm{ca}}$ loops are different. Although the distortion may occur in three phase currents, the level of distortion can be lessened by using coupled inductors instead, or raising the effective switching frequency.

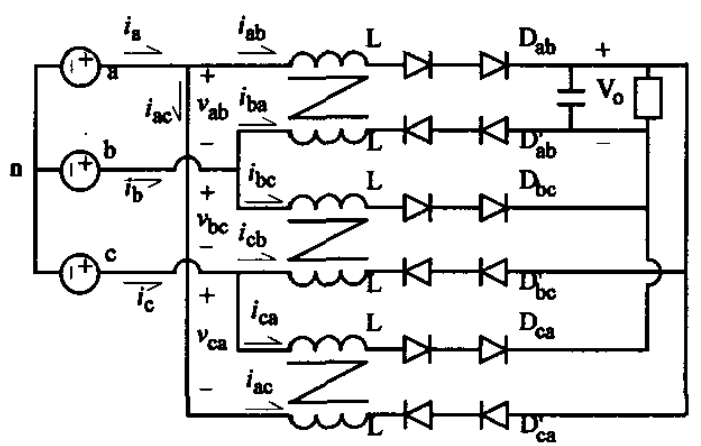

Fig. 5 The conduction paths when $S_{a b}, S_{b c}$ and $S_{c a}$ are turned off.

Since each phase current is the sum of two line currents, the phase current will be affected by the states of the two switches which belong to the two loops containing the referred phase voltage. For example, the ripple of $i_{\mathrm{a}}$ depends on the switching states of $S_{a b}$ and $S_{c a}$. In the worst case it can be twice the width of the defined hysteresis band. The block diagram of the proposed threephase system is illustrated in Fig. 6 . The single output voltage feedback loop produces the same current command for three single-phase SMR's. Thus nearly equal current sharing among three phases is guaranteed. It is noted that the neutral potential is never used in the controller. We sense the line currents and the line-to-line voltages instead of the phase currents and the phase voltages. The access of the neutral is unnecessary. 


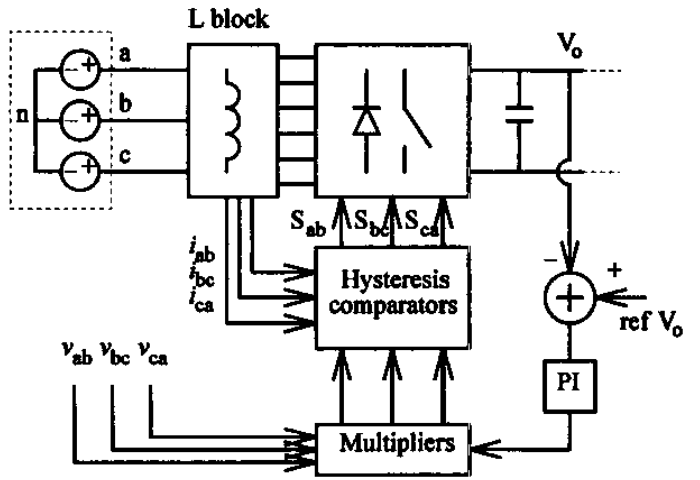

Fig. 6 The block diagram of the proposed threewire Y-connected system.

\section{Simulations and Experiments}

Simulations are performed to verify the proposed three-phase SMR. Fig. 7 depicts the phase-a voltage and current. In Fig. 8, input currents of three phases are shown. It can be seen that the ripples of each phase current are not constant, which agrees with the results discussed earlier. Fig. 9(a) shows the current imbalance occured in $v_{\mathrm{ab}}$ loop with two separate inductors due to the interactions among three phases. In Fig. 9(b), it can be observed that the interaction level is greatly reduced by using coupled inductors instead.

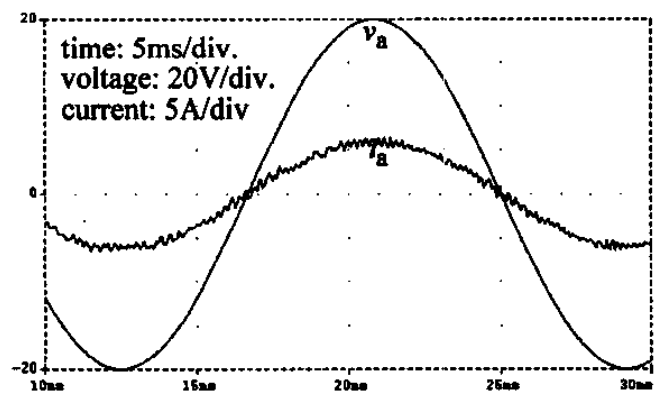

Fig. 7 The simulated phase voltage and current of phase-a.

Experiments are conducted on a three-phase propotype. To get the phase voltages for comparison, here we use a four-wire Y-connected source for the access of the neutral, but otherwise the system arrangement is unchanged. In Fig. 10, the input current of phase-a is recorded. Fig. 11 shows the phase currents of three phases under unity power factor control.

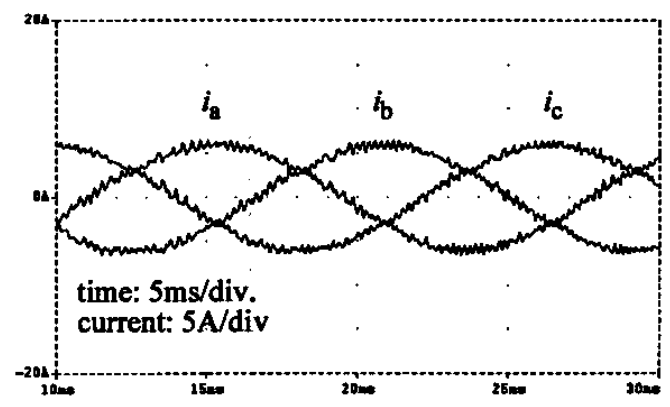

Fig. 8 The simulated three phase currents.

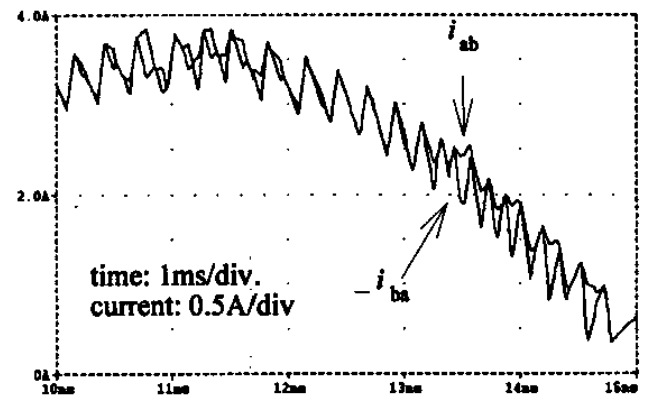

(a)

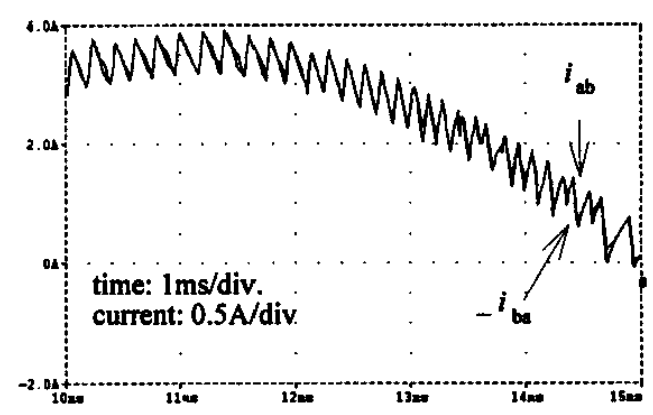

(b)

Fig. 9 Simulated $i_{\mathrm{ab}}$ and $-i_{\mathrm{ba}}$ with (a) decoupled inductors and (b) coupled inductors. 


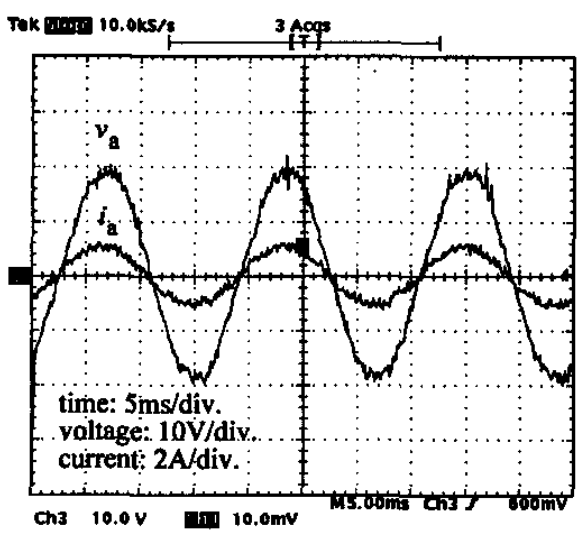

Fig. 10 The phase voltage and current of phase-a.

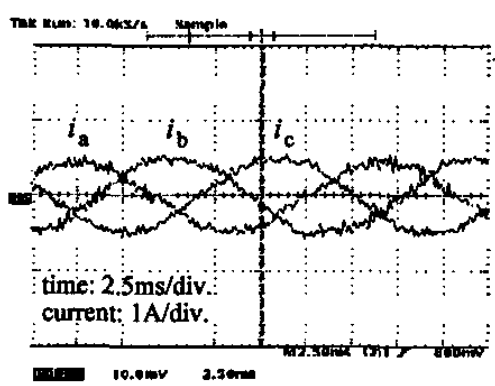

Fig. 11 The input currents of three phases.

Fig. 12(a) depicts the current imbalance between $i_{\mathrm{ab}}$ and $-i_{\mathrm{ba}}$ with two separate inductors when $S_{\mathrm{ab}}$ and at least one of the other two switches are turned off. On the top is the gate signal for $\mathrm{S}_{\mathrm{ab}}$. Fig. 12(b) shows that the imbalance has been improved by using coupled inductors instead.

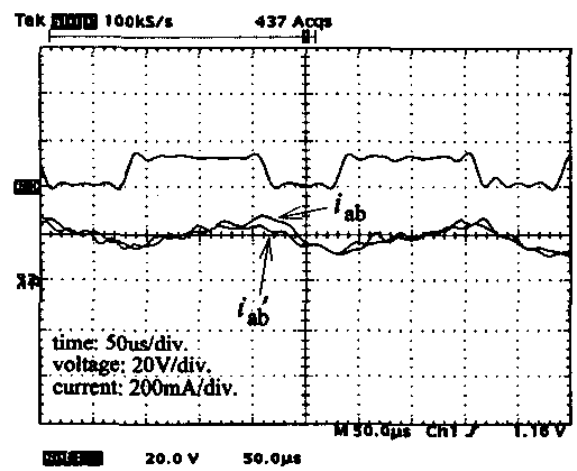

(a)

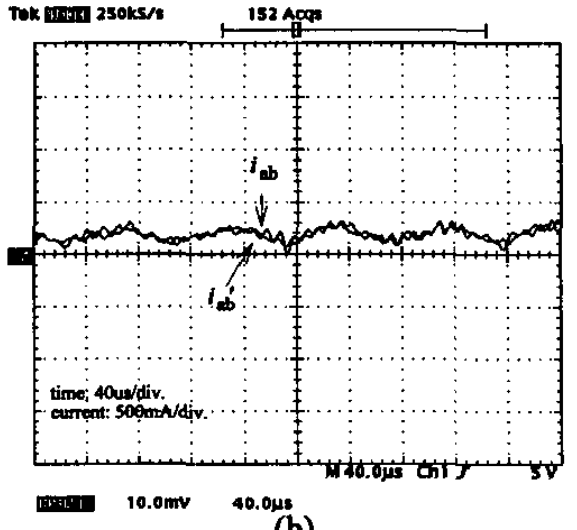

(b)

Fig. $12 i_{\mathrm{ab}}$ and $-i_{\mathrm{ba}}$ with (a) decoupled inductors and (b) coupled inductors.

\section{Conclusions}

In this paper, a three-phase switch-mode rectifier (SMR) for three-wire Y-connected sources with an unaccessible neutral is proposed. Each phase current is split into two line currents, which are controlled to be sinusoidal and in-phase with the corresponding line-to-line voltages. There is no need for the access of the neutral. The results show that the coupling among different stages can be lessened. Totally there are 12 diodes and 6 fast-recovery diodes needed. However, only 3 active switches are used and dead-time insertion is no longer required. The control mechanism is less complex than a high-performance 6-switch bridge topology.

\section{References}

[1] B. T. OOi, J. C. Salmon, J. W. Dixon, and A. B. Kulkarni, "A Three-Phase Controlled Current PWM Converter with Leading Power Factor," in IEEE Trans. Ind. Applica., vol. IA-23, no. 1, pp.252-256, Jan./Feb. 1987.

[2] M. S. Dawande, V. R. Kanetkar, and G. K. Dubey, "Three-Phase Switch Mode Rectifier with Hysteresisi Current Control," IEEE Trans. Power Electron., vol. PE-11, no. 3, pp.466-471, May 1996.

[3] G. Spiazzi, and F. C. Lee, "Implementation of Single-Phase Boost Power-Factor-Correction Circuits in Three-Phase Applications," IEEE Trans. Ind. Electron., vol. IE-44, no. 3, pp. 365-371,June 1997. 